\begin{tabular}{|c|l|}
\hline Title & Spatial and Temporal Growth of Perturbations in Open-Channel Viscous Transverse Shear Flow \\
\hline Author(s) & de Lima, A driano C.; Izumi, Norihiro \\
\hline Citation & $\begin{array}{l}\text { Journal of hydraulic engineering, 142(12), 04016067 } \\
\text { https://doi.org/10.1061/A SCE)HY.1943-7900.0001220 }\end{array}$ \\
\hline Issue Date & 2016-12 \\
\hline Doc URL & http://hdl.handle.net/2115/64032 \\
\hline Type & article (author version) \\
\hline Note(URL) & http://ascelibrary.org/doi/10.1061\% 28A SCE\% 29HY.1943 7900.0001220 \\
\hline File Information & ascexmpl.pdf \\
\hline
\end{tabular}

Instructions for use 


\title{
SPATIAL AND TEMPORAL GROWTH OF PERTURBATIONS IN OPEN-CHANNEL VISCOUS TRANSVERSE SHEAR FLOW
}

\author{
Adriano C. de Lima ${ }^{1}$, Norihiro Izumi ${ }^{2}$
}

\begin{abstract}
The comparison between spatial and temporal growth of perturbations in a shear layer is revisited for the case of a viscous open-channel flow with velocity inflection. Turbulence is characterized by two distinct scales: a sub-depth turbulence associated with the bed shear stress and a large-scale turbulence associated with the large horizontal eddies which develop in the shear layer. Temporal and spatial linear stability analyses are performed. The base flow is assumed to be affected only by the sub-depth scale turbulence. Therefore, the base flow represents not the average flow but the flow completely free of the effect of the instability of the shear layer. Temporal and spatial approaches present less discrepancy in the viscous than in the previously analyzed inviscous case. In particular, the phase velocity was found to be strongly correlated to the frequency in both the temporal and spatial approaches.
\end{abstract}

Keywords: Spatial and temporal linear stability analysis, open-channel viscous flow, shear layer instability, kinematic eddy viscosity.

\section{INTRODUCTION}

Geophysical flows with velocity inflection and transverse shear are observed, for example, in composite channels, in inflows at channel junctions, in channels where the bottom roughness varies laterally, or in channels with lateral vegetation or pile dikes (Tamai et al. 1986; Chu et al. 1991; Ikeda et al. 1991; Ikeda et al. 1994; Tsujimoto and Kitamura 1995; Nadaoka and Yagi 1998; Uijttewaal and Booij 2000; Su and Li 2002; Prooijen and Uijttewaal 2002; White and Nepf 2007).

\footnotetext{
${ }^{1}$ Division of Field Engineering for the Environment, Graduate School of Engineering, Hokkaido University, Sapporo, Japan, 0608628. E-mail: adriano@eng.hokudai.ac.jp.

${ }^{2}$ Division of Field Engineering for the Environment, Graduate School of Engineering, Hokkaido University, Sapporo, Japan, 0608628. E-mail: nizumi@eng.hokudai.ac.jp.
} 
Transverse shear results in a Kelvin-Helmholtz instability with large-scale horizontal vortices centered around the velocity inflection point (Ikeda et al. 1994; Prooijen and Uijttewaal 2002; White and Nepf 2007). These vortices have a strong influence on the velocity distribution and the amount of discharge conveyed by a channel without overflow, and enhance the lateral mixing of not only the flow itself, but also the substances transported by the flow. Precise estimations of discharge capacity, velocity distribution and shear distribution in channels and rivers are required for practical engineering problems such as river restoration, flood reduction measures, flood plain management, control of sediment and pollutant transport in rivers, and aquatic habitat evaluation (Uijttewaal and Booij 2000; Kolyshkin and Ghidaoui 2002; Carnie et al. 2015).

Stability characteristics of transverse shear layers have been studied extensively by means of linear stability analysis. The inviscid flow assumption was employed in the early works of Michalke (1964, 1965) and then by e.g. Monkewitz and Huerre (1982), Tamai et al. (1986), Chu et al. (1991), Ikeda et al. (1994) and White and Nepf (2007). Under the inviscid flow assumption, the perturbation equations in these studies are reduced to modified Rayleigh equations. On the other hand, the formulations with turbulence viscosity, such as those of Prooijen and Uijttewaal (2002), Ghidaoui and Kolyshkin (1999), Kolyshkin and Ghidaoui (2002), Chen and Jirka (1997) and Lima and Izumi (2014a, 2014b), lead to modified Orr-Sommerfeld equations. The inviscid flow assumption is a reasonable simplification for flows at large Reynolds numbers or flows where the changes in the depth, bottom roughness or vegetation density across the channel are gradual.

In most of the previous works mentioned above a stability analysis was performed for the temporal case, in which the disturbance is formulated as timely-growing, with the exception of Michalke (1965), Monkewitz and Huerre (1982) and White and Nepf (2007), who performed the analysis for the spatial case of an inviscid flow. It is widely acknowledged that the results of the spatial stability analysis tend to be in better agreement with experimental data than those from the temporal stability analysis. However, the spatial stability analysis of viscous flows leads to a nonlinear eigenvalue problem which is more complicated to solve than the linear eigenvalue problem resulting from the temporal approach. 
In the present work, spatial and temporal linear stability analyses are performed for the case of a viscous shear layer in an open-channel flow. The flow is formulated with the use of the St. Venant shallow water equations with the Reynolds stress expressed by a kinematic eddy viscosity representing turbulence with a length scale smaller than the flow depth (Lima and Izumi 2014a). This is different from previous formulations with turbulence viscosity (Prooijen and Uijttewaal 2002; Ghidaoui and Kolyshkin 1999) where the Reynolds stress is modeled employing a kinematic eddy viscosity which is affected by the lateral motions due to the shear layer.

Results from the spatial and temporal analysis are compared, and the differences among both approaches are found to be less relevant than those from Michalke (1965). The perturbed velocity cross-distribution and the phase and group velocities determined for the temporal case in the present work are particularly distinct from those from Michalke (1965).

\section{CONCEPTUAL MODEL AND ANALYTICAL FORMULATION}

The formulation employed in the present work follows that of Lima and Izumi (2014a, 2014b), who performed stability analyses for the temporal case. The application of this formulation to the growth of disturbances in space is novel. A wide rectangular open-channel flow with an inflection point in the depth-averaged streamwise velocity is considered. It is assumed that the velocity gradient is due to the presence of lateral emergent rigid vegetation (pile dikes), as depicted in Fig. 1. The vegetation is modeled by an array of regularly spaced cylinders with a uniform diameter installed only on one side of the channel, a set up which has been widely used in previous works (e.g., Ikeda et al. (1991), Tsujimoto (1991), Ikeda et al. (1994), Kitamura et al. (1998), Su and Li (2002), White and Nepf $(2007,2008))$. The streamwise coordinate is denoted by $\tilde{x}$ and the transverse coordinate is denoted by $\tilde{y}$, the origin of which being taken at the interface between the non-vegetated and vegetated zones, assuming positive values in the former. The widths of the non-vegetated and vegetated zones are denoted by $\tilde{B}$ and $\tilde{B}_{v}$, respectively. The channel bottom has a constant slope along its streamwise direction denoted by $S$. The rigid-lid assumption is not employed.

The horizontal length scale of the vortices is commonly large compared with the scale of the 
flow depth. The generation of such thin vortices can be described by the depth-averaged shallow water formulation. The momentum and the continuity equations are then introduced in the form

$$
\begin{aligned}
\frac{\partial \overline{\boldsymbol{U}}}{\partial \tilde{t}}+(\overline{\tilde{\boldsymbol{U}}} \cdot \tilde{\nabla}) \overline{\tilde{\boldsymbol{U}}} & =\tilde{\boldsymbol{g}}-\tilde{g} \tilde{\boldsymbol{\nabla}} \tilde{\tilde{H}}-\frac{\overline{\boldsymbol{T}}_{b}+\overline{\tilde{\boldsymbol{D}}}}{\tilde{\rho} \overline{\tilde{H}}}+\tilde{\nu}_{T} \tilde{\nabla}^{2} \tilde{\tilde{\boldsymbol{U}}} \\
\frac{\partial \tilde{\tilde{H}}}{\partial \tilde{t}}+\tilde{\nabla} \cdot(\overline{\tilde{H} \tilde{\boldsymbol{U}}}) & =0
\end{aligned}
$$

where $\tilde{t}$ is time, $\tilde{\boldsymbol{U}}=(\tilde{U}, \tilde{V})$ is the velocity vector with components $\tilde{U}$ and $\tilde{V}$ at the $\tilde{x}$ and $\tilde{y}$ directions, respectively, $\tilde{\boldsymbol{T}}_{b}$ is the bed shear stress vector, $\tilde{\boldsymbol{D}}$ is the vegetation drag stress vector, $\tilde{\boldsymbol{g}}=(\tilde{g} S, 0)$ is the gravity vector, where $\tilde{g}$ is the gravity acceleration and $S$ is the bed slope, $\tilde{H}$ is the flow depth, $\tilde{\rho}$ is the density of the fluid, $\tilde{\nu}_{T}$ is the kinematic eddy viscosity, $\tilde{\nabla}=\left(\frac{\partial}{\partial \tilde{x}}, \frac{\partial}{\partial \tilde{y}}\right)$ and $\tilde{\nabla}^{2}=\tilde{\nabla} \cdot \tilde{\nabla}$. The tilde denotes dimensional variables and the overline denotes variables averaged over a short time scale corresponding with the sub-depth scale turbulences. The overline is dropped afterwards for simplicity.

The bed shear stress is related to the flow velocity by means of the bed friction coefficient $C_{f}$, such that

$$
\tilde{\boldsymbol{T}}_{b}=\left(\tilde{T}_{b x}, \tilde{T}_{b y}\right)=\tilde{\rho} C_{f}\left(\tilde{U}^{2}+\tilde{V}^{2}\right)^{1 / 2}(\tilde{U}, \tilde{V})
$$

where $\tilde{T}_{b x}$ and $\tilde{T}_{b y}$ are the $\tilde{x}$ and $\tilde{y}$ components of the bed shear stress vector $\tilde{\boldsymbol{T}}_{b}$, respectively. Though the bed friction coefficient $C_{f}$ is a weak function of the flow depth relative to the roughness height, it is assumed to be constant and common in both the vegetated and non-vegetated zones for simplicity.

The vegetation drag stress vector $\tilde{D}$ is described by the expression

$$
\tilde{\boldsymbol{D}}=\left(\tilde{D}_{x}, \tilde{D}_{y}\right)= \begin{cases}0 & \text { if } \quad 0 \leq \tilde{y} \leq \tilde{B} \\ \frac{\tilde{\rho} C_{d} \tilde{a} \tilde{H}}{2}\left(\tilde{U}^{2}+\tilde{V}^{2}\right)^{1 / 2}(\tilde{U}, \tilde{V}) & \text { if } \quad-\tilde{B}_{v} \leq \tilde{y} \leq 0\end{cases}
$$

where $\tilde{D}_{x}$ and $\tilde{D}_{y}$ are the components of the vector $\tilde{\boldsymbol{D}}$ in the $\tilde{x}$ and $\tilde{y}$ directions, respectively, $C_{d}$ is the drag coefficient of vegetation, typically estimated to range from 1 to 2 , and $\tilde{a}=\tilde{d} /\left(2 \tilde{l}_{x} \tilde{l}_{y}\right)$ is the 
parameter which describes the density of vegetation, where $\tilde{d}$ is the diameter of the cylinders and $\tilde{l}_{x}$ and $\tilde{l}_{y}$ are the distances between the center of two adjacent cylinders in the $\tilde{x}$ and $\tilde{y}$ directions, respectively, as shown in Figure 1.

It is assumed that, in the base state before instability occurs, the flow is already affected by turbulence, which would be smaller in the length scale than the flow depth (sub-depth scale turbulence). Where there is no influence of vegetation, the kinematic eddy viscosity $\tilde{\nu}_{T}$ should correspond to the sub-depth scale turbulence generated by the bottom friction. The logarithmic velocity distribution is employed to represent the sub-depth scale turbulent velocity distribution affected by the bottom friction. The kinematic eddy viscosity then takes a parabolic form, which is depthaveraged from the bottom to the water surface, yielding (Fischer et al. 1979; Lima and Izumi 2014a)

$$
\tilde{\nu}_{T}=\frac{1}{6} \kappa \tilde{U}_{f} \tilde{H}_{\infty}
$$

where $\tilde{U}_{f}\left(=\sqrt{\tilde{T}_{b x \infty} / \tilde{\rho}}\right)$ and $\tilde{H}_{\infty}$ are the friction velocity and the flow depth in the region sufficiently far from the vegetated zone (denoted by the subscript $\infty$ ), respectively, and $\kappa$ is the Kármán constant $(=0.4)$. The sub-depth scale turbulence is assumed as isotropic. Therefore, the above formulation is expected to describe the Reynolds stresses also in the streamwise and lateral directions at a sufficient distance from the vegetated zone.

In the shear layer formed around the boundary between the two zones, and inside the vegetated zone, the velocity and the shear velocity are reduced because of the Reynolds stress and the drag force due to vegetation. In addition, the length scale of sub-depth scale vortices may be affected by a typical length scale of vegetation, such as the spacing of the vegetation. According to the experimental results of Ikeda et al. (1991), however, the depth-averaged kinematic eddy viscosity even in the shear layer and the vegetated zone can be represented by equation (4). This may be attributed to the fact that the sum of the resistant forces (the bed shear stress, the Reynolds stress and the vegetation drag force) remains constant regardless of the reduction in the bed shear stress in the shear layer and the vegetated zone. The kinematic eddy viscosity may be correlated to the total resistant force. Furthermore, since the flow depth and the spacing of vegetation in the experiments 
carried out by Ikeda et al. (1991) are both in the same range, it may well be that the kinematic eddy viscosity in the vegetated zone is not strongly affected by vegetation. These assumptions and (4) are employed in this study as well.

Fischer et al. (1979) determined the transverse mixing coefficient $\varepsilon_{t} \cong 0.15 \tilde{U}_{f} \tilde{H}$ from an approximate average of experimental results in straight rectangular channels from various studies, stating that the above result is likely to be correct for practical purposes in straight rectangular channels with an error bound of $\pm 50 \%$. Based on Fischer et al. (1979), Chen and Jirka (1997), followed by Ghidaoui and Kolyshkin (1999) and Prooijen and Uijttewaal (2002), have assumed values of kinematic eddy viscosity in the range $\tilde{\nu}_{t}=0.15-0.20 \tilde{U}_{f} \tilde{H}$ for expressing the Reynolds stress corresponding to the small-scale motions. This range of values of kinematic eddy viscosity is, however, affected by the transverse mixing of the shear layer. On the other side, the eddy viscosity in equation (4) is estimated for the flow without the transverse mixing generated by the shear layer. Thus, it assumes a value smaller than $\tilde{\nu}_{t}=0.15-0.20 \tilde{U}_{f} \tilde{H}$. By employing (4) in the formulation, a base flow consisting of the flow completely undisturbed by the lateral motions due to the development of the shear layer can be derived.

The channel is assumed to be sufficiently wide to accommodate a shear layer which does not reach the side walls. Under this assumption, it follows that the transverse velocity $\tilde{V}$ vanishes and the streamwise velocity $\tilde{U}$ asymptotically approaches to constant slip velocities at the side walls of the channel. The following boundary conditions therefore hold:

$$
\tilde{V}=0 \quad \text { at } \quad \tilde{y}=-\tilde{B}_{v}, \tilde{B}
$$

$$
\frac{\partial \tilde{U}}{\partial \tilde{y}}=0 \quad \text { at } \quad \tilde{y}=-\tilde{B}_{v}, \tilde{B}
$$

Right at the boundary between the non-vegetated and vegetated zones, the velocities, the flow depth and the shear stresses are continuous, such that the following matching conditions hold:

$$
\lim _{\tilde{y} \rightarrow+0}\left(\tilde{\boldsymbol{U}}, \frac{\partial \tilde{\boldsymbol{U}}}{\partial \tilde{y}}, \tilde{H}\right)=\lim _{\tilde{y} \rightarrow-0}\left(\tilde{\boldsymbol{U}}, \frac{\partial \tilde{\boldsymbol{U}}}{\partial \tilde{y}}, \tilde{H}\right)
$$


The above conditions are valid provided the vegetation is not sufficiently dense for a stress jump to take place at $\tilde{y}=0$.

It is assumed that at a sufficient distance from the boundary between the non-vegetated and vegetated zones, the $\tilde{x}$-direction derivatives vanish, along with the $\tilde{y}$ direction velocity component $\tilde{V}$. This yields a constant flow depth $\tilde{H}_{0}=\tilde{H}_{\infty}$. At $\tilde{y}=\tilde{B}$ and $y=-\tilde{B}_{v}$, the far field velocities, denoted as $\tilde{U}_{\infty}$ and $\tilde{U}_{-\infty}$, respectively, take the form

$$
\tilde{U}_{\infty}=\left(\frac{\tilde{g} \tilde{H}_{\infty} S}{C_{f}}\right)^{1 / 2}, \quad \tilde{U}_{-\infty}=\left(\frac{2 \tilde{g} \tilde{H}_{\infty} S}{2 C_{f}+C_{d} \tilde{a} \tilde{H}_{\infty}}\right)^{1 / 2}
$$

where the subscript $-\infty$ denotes the far field in the vegetated zone. In experiments, the bed friction coefficient $C_{f}$ and the vegetation drag coefficient $C_{d}$ can be determined from the above equations once $\tilde{U}_{\infty}, \tilde{U}_{-\infty}$ and $\tilde{H}_{\infty}$ are measured.

The velocity $\tilde{U}_{\infty}$, the flow depth $\tilde{H}_{0}$ and the non-vegetated zone with $\tilde{B}$ are taken as the characteristic velocity, vertical length scale and horizontal length scale, respectively, for normalization, which is introduced in the form

$$
\tilde{\boldsymbol{U}}=\tilde{U}_{\infty} \boldsymbol{U}, \quad \tilde{H}=\tilde{H}_{0} H, \quad\left(\tilde{x}, \tilde{y}, \tilde{B}_{v}\right)=\tilde{B}\left(x, y, B_{v}\right), \quad \tilde{t}=\frac{\tilde{B}}{\tilde{U}_{\infty}} t
$$

With the use of the above normalization expressions, the governing equations (1) are rewritten in the form

$$
\begin{aligned}
\frac{\partial \boldsymbol{U}}{\partial t}+(\boldsymbol{U} \cdot \boldsymbol{\nabla}) \boldsymbol{U} & =-F^{2} \boldsymbol{\nabla} H+\beta\left(\boldsymbol{g}-\frac{\boldsymbol{T}_{b}+\boldsymbol{D}}{H}\right)+\epsilon \nabla^{2} \boldsymbol{U} \\
\frac{\partial H}{\partial t}+\nabla \cdot(H \boldsymbol{U}) & =0
\end{aligned}
$$

where $F$ is the Froude number, $\beta$ is the bed-friction parameter and $\epsilon$ is the sub-depth kinematic eddy viscosity parameter, expressed in the forms

$$
F=\frac{\tilde{U}_{\infty}}{\sqrt{\tilde{g} \tilde{H}_{0}}}=\left(\frac{S}{C_{f}}\right)^{1 / 2}, \quad \beta=\frac{C_{f} \tilde{B}}{\tilde{H}_{0}}, \quad \epsilon=\frac{\tilde{\nu}_{T}}{\tilde{U}_{\infty} \tilde{B}}=\frac{C_{f}^{1 / 2} \tilde{H}_{0}}{15 \tilde{B}}
$$


The non-dimensional gravity vector $\boldsymbol{g}$, bed shear stress vector $\boldsymbol{T}_{b}$ and vegetation drag vector $\boldsymbol{D}$ take the forms:

$$
\begin{aligned}
& \boldsymbol{g}=(1,0), \\
& \boldsymbol{T}_{b}=\left(T_{b x}, T_{b y}\right)=\left(U^{2}+V^{2}\right)^{1 / 2}(U, V), \\
& \boldsymbol{D}=\left(D_{x}, D_{y}\right)=\left\{\begin{array}{ccc}
0 & \text { if } & 0 \leq y \leq 1, \\
\alpha\left(U^{2}+V^{2}\right)^{1 / 2} H(U, V) & \text { if } & -B_{v} \leq y \leq 0,
\end{array}\right.
\end{aligned}
$$

where the non-dimensional parameter $\alpha$ is related to the vegetation drag and density, and is defined by

$$
\alpha=\frac{C_{d} \tilde{a} \tilde{H}_{0}}{2 C_{f}} .
$$

\section{Asymptotic expansions and the base flow solution}

The flow velocity components $U$ and $V$ and the flow depth $H$ are decomposed into a base state of order $O(1)$ and a perturbed component of order $O(A)$ in the form:

$$
\left(\begin{array}{c}
U(x, y, t) \\
V(x, y, t) \\
H(x, y, t)
\end{array}\right)=\left(\begin{array}{c}
U_{0}(y) \\
0 \\
1
\end{array}\right)+A\left(\begin{array}{c}
U_{1}(y) \\
V_{1}(y) \\
H_{1}(y)
\end{array}\right) \mathrm{e}^{\mathrm{i}(k x-\omega t)},
$$

where $A, k$ and $\omega$ are the amplitude, wavenumber and angular frequency of the disturbance introduced to the base flow, respectively.

The base state flow field is set as the flow undisturbed by the horizontal vortices. Because it has only a streamwise component, it follows that the time and space derivatives and the transverse velocity vanish and the flow depth is equal to unity. Substituting the terms of order $O(1)$ from (13) into (9) yields

$$
\begin{aligned}
& \beta\left(1-U_{0}^{2}\right)+\epsilon \frac{\mathrm{d}^{2} U_{0}}{\mathrm{~d} y^{2}}=0 \quad \text { if } \quad 0 \leq y \leq 1, \\
& \beta\left[1-U_{0}^{2}(1+\alpha)\right]+\epsilon \frac{\mathrm{d}^{2} U_{0}}{\mathrm{~d} y^{2}}=0 \quad \text { if } \quad-B_{v} \leq y \leq 0 .
\end{aligned}
$$


The analytical solution of (14) under the normalized forms of the boundary and matching conditions (5)-(6) yields the following explicit expressions of $U_{0}$ :

$$
U_{0}(y)= \begin{cases}3 \tanh ^{2}\left[\left(\frac{\beta}{2 \epsilon}\right)^{1 / 2} y+\tanh ^{-1}\left(\frac{\psi+2}{3}\right)^{1 / 2}\right]-2 & \text { if } \quad 0 \leq y \leq 1, \\ 3 \phi \operatorname{coth}^{2}\left[-\left(\frac{\beta}{2 \epsilon \phi}\right)^{1 / 2} y+\operatorname{coth}^{-1}\left(\frac{\psi+2 \phi}{3 \phi}\right)^{1 / 2}\right]-2 \phi & \text { if }-B_{v} \leq y \leq 0,\end{cases}
$$

where $\phi$ is the normalized base flow velocity in the vegetated zone sufficiently far from the interface with the non-vegetated zone and $\psi$ is the normalized base flow velocity at the interface between the non-vegetated and vegetated zones $(y=0)$. The parameters $\phi$ and $\psi$ are explicit functions of the vegetation drag and density parameter $\alpha$ in the forms:

$$
\phi=\frac{1}{(1+\alpha)^{1 / 2}}, \quad \psi=\left[\frac{2}{1+\alpha+(1+\alpha)^{1 / 2}}\right]^{1 / 3} .
$$

The domain of $\phi$ is $0<\phi \leq 1 ; \phi$ approaches 0 when the vegetation obstructs the flow completely in the vegetated zone $(\alpha \rightarrow \infty)$, and takes a value of unity when there is no vegetation $(\alpha=0)$.

The base flow velocity $U_{0}$ is expressed by squared hyperbolic-tangent and squared hyperboliccotangent functions which are invariant in time and in the streamwise direction and include four non-dimensional parameters, $\beta, \epsilon, \psi$ and $\phi$, where the latter two can be expressed as functions of only $\alpha$. Fig. 2 depicts sample base state velocity profiles for various values of the eddy viscosity parameter $\epsilon$. The velocities at the far right and left correspond to $\tilde{U}_{\infty}$ and $\tilde{U}_{-\infty}$ respectively, and the value of $U_{0}$ at the far left is $\phi$.

The base flow assumption herein consists of the flow undisturbed by the large scale vortices, which is remarkably distinct from previous approaches (e.g. Michalke (1964, 1965), Chu et al. (1991), Prooijen and Uijttewaal (2002), White and Nepf (2007)) where the base flow is set as the average flow and for which the velocity distribution is fit to a hyperbolic tangent profile. The lateral distributions of the base flow velocity $U_{0}$ and the normalized measured streamwise velocity $U$ at the developed state of the perturbations are depicted in Fig. 3 for run 1 of the experiments 
of Ikeda et al. (1991), where vortices were generated in an open-channel featuring a lateral array of regularly spaced cylinders, which is the same set-up assumed in the present formulation. As expected, the shear layer is narrower in the base flow rather than in the developed average flow. The shear layer widens as the perturbations grow nonlinearly. This study is, however, limited to the linear reach of the perturbation development.

The velocity reduction in the boundary layer at the vicinity of the side walls, clearly visualized in Fig. 3 at the far field in the non-vegetated zone, is not considered in the present analytical model because it would virtually not affect the shear layer, since the channel is considered to be sufficiently wide.

\section{LINEAR STABILITY ANALYSIS}

The substitution of (13) into the normalized governing equations (9) yields the following perturbed equations in the non-vegetated zone:

$$
\begin{aligned}
{\left[\mathrm{i}\left(k U_{0}-\omega\right)+k^{2} \epsilon+2 \beta U_{0}-\epsilon \frac{\mathrm{d}^{2}}{\mathrm{~d} y^{2}}\right] U_{1}+\frac{\mathrm{d} U_{0}}{\mathrm{~d} y} V_{1}+\left(\mathrm{i} k F^{-2}-\beta U_{0}^{2}\right) H_{1} } & =0 \\
{\left[\mathrm{i}\left(k U_{0}-\omega\right)+k^{2} \epsilon+\beta U_{0}-\epsilon \frac{\mathrm{d}^{2}}{\mathrm{~d} y^{2}}\right] V_{1}+F^{-2} \frac{\mathrm{d} H_{1}}{\mathrm{~d} y} } & =0 \\
\mathrm{i} k U_{1}+\frac{\mathrm{d} V_{1}}{\mathrm{~d} y}-\mathrm{i}\left(\omega-k U_{0}\right) H_{1} & =0
\end{aligned}
$$

and the following perturbed equations in the vegetated zone,

$$
\left[\mathrm{i}\left(k U_{0}-\omega\right)+k^{2} \epsilon+2 \beta U_{0}(1+\alpha)-\epsilon \frac{\mathrm{d}^{2}}{\mathrm{~d} y^{2}}\right] U_{1}+\frac{\mathrm{d} U_{0}}{\mathrm{~d} y} V_{1}+\left(\mathrm{i} k F^{-2}-\beta U_{0}^{2}\right) H_{1}=0,
$$

$$
\left[\mathrm{i}\left(k U_{0}-\omega\right)+k^{2} \epsilon+\beta U_{0}(1+\alpha)-\epsilon \frac{\mathrm{d}^{2}}{\mathrm{~d} y^{2}}\right] V_{1}+F^{-2} \frac{\mathrm{d} H_{1}}{\mathrm{~d} y}=0,
$$

$$
\mathrm{i} k U_{1}+\frac{\mathrm{d} V_{1}}{\mathrm{~d} y}-\mathrm{i}\left(\omega-k U_{0}\right) H_{1}=0 .
$$


Because the amplitude of the perturbations $A$ is assumed to be infinitesimally small in the scheme of linear stability analysis, terms containing $A^{2}$ were dropped from (17-18).

A numerical scheme is necessary to solve (17-18) under the corresponding expanded forms of the boundary and matching conditions (5-6), as the resulting system of equations obviously does not allow analytical solutions. A spectral collocation method with the Chebyshev polynomials is employed. In the non-vegetated zone $(0 \leq y \leq 1)$, the variables $U_{1}, V_{1}$ and $H_{1}$ are expanded in the form

$$
U_{1}=\sum_{j=0}^{N} a_{j} T_{j}(\xi), \quad V_{1}=\sum_{j=0}^{N} a_{(N+1)+j} T_{j}(\xi), \quad H_{1}=\sum_{j=0}^{N} a_{2(N+1)+j} T_{j}(\xi),
$$

and in the vegetated zone $\left(-B_{v} \leq y \leq 0\right)$, these variables are expanded in the form

$$
U_{1}=\sum_{j=0}^{N} a_{3(N+1)+j} T_{j}(\gamma), \quad V_{1}=\sum_{j=0}^{N} a_{4(N+1)+j} T_{j}(\gamma), \quad H_{1}=\sum_{j=0}^{N} a_{5(N+1)+j} T_{j}(\gamma)
$$

where $a_{j}(j=0,1,2, \ldots, 6 N+5)$ are the coefficients of the Chebyshev polynomials, and $T_{j}(\xi)$ and $T_{j}(\gamma)$ are the Chebyshev polynomials in $\xi$ and $\gamma$ of degree $j$ respectively. The independent variables $\xi$ and $\gamma$ both range from -1 to 1 , and are related to $y$ by the equations $\xi=2 y-1$ $(0 \leq y \leq 1)$ and $\gamma=2 y / B_{v}+1\left(-B_{v} \leq y \leq 0\right)$, respectively. The expansions (19) and (20) are substituted into the governing equations (17) and (18) respectively, and the resulting six equations are evaluated at the Gauss-Lobatto points defined by

$$
\xi_{m}=\cos \frac{m \pi}{N}, \quad \gamma_{m}=\cos \frac{m \pi}{N},
$$

where $m=0,1, \ldots, N$. Therefore, the number of points where the governing equations are evaluated is $N+1$. A system of $6(N+1)$ algebraic equations with $6(N+1)$ unknown coefficients $a_{0}, a_{1}, a_{2}, \ldots, a_{6 N+5}$ is obtained. Eight equations of the system are then replaced by the four boundary conditions (5) and four of the matching conditions (6). The resulting linear algebraic system takes the form

$$
\mathcal{M A}=0
$$


where $\mathcal{M}$ is a $6(N+1) \times 6(N+1)$ matrix in which the elements are derived from the coefficients of $U_{1}, V_{1}$ and $H_{1}$ in the governing equations (17-18) and the boundary and matching conditions (5-6), and $\boldsymbol{A}=\left[a_{0}, a_{1}, \ldots, a_{6 N+5}\right]^{T}$. The condition for (22) to have a non-trivial solution is that $\mathcal{M}$ should be singular. Thus,

$$
|\mathcal{M}|=0
$$

Under the temporal framework of stability analysis, it is assumed that, in the present set up, the domain of the streamwise direction $\tilde{x}$ is sufficiently long to contain one or more complete periods of a spatially periodic disturbance. The amplitude of this disturbance grows as time progresses. Under the spatial framework, on the other hand, the disturbances are assumed to be generated at a fixed position which, in the present set up, would correspond to the upstream boundary condition of the partially vegetated channel. And the amplitude of the disturbances grows in the downstream direction (Schmid and Henningson (2001)).

Although the spatial analysis is more complex because the eigenvalue appears nonlinearly, there are physical situations, such as harmonic point sources, which require the modeling of the disturbance amplitude as a spatially growing quantity (Schmid and Henningson (2001)). For mixing layers, both temporal and spatial approaches have led to reasonable results, though only the latter has been regarded for accounting for cross-stream distributions, in the case of an inviscid hyperbolic-tangent base flow (Michalke (1964), Michalke (1965), Freymuth (1966), Ho and Huerre (1984)), which is distinct from the base flow in the present work.

In the scheme of temporal linear stability analysis, the wavenumber is real, and is denoted as $k^{t}=k_{r}^{t}$, while the angular frequency is complex such that $\omega^{t}=\omega_{r}^{t}+\mathrm{i} \omega_{i}^{t}$, where $\omega_{r}^{t}$ is the real angular frequency and $\omega_{i}^{t}$ is the growth rate of the disturbance in time. As time progresses, the perturbation grows if $\omega_{i}^{t}>0$ and decays if $\omega_{i}^{t}<0$. In the temporal analysis, it is convenient to decompose the matrix $\mathcal{M}$ in the form

$$
\mathcal{M}=\mathcal{L}^{t}+\omega^{t} \mathcal{R}^{t}
$$


where the entries of $\mathcal{L}^{t}$ are composed by the terms of the entries of $\mathcal{M}$ which do not contain $\omega^{t}$, and the entries of $\mathcal{R}^{t}$ are composed by the coefficients of $\omega^{t}$ in the entries of $\mathcal{M}$. Substituting the above decomposition into (22) yields the following generalized eigenproblem:

$$
\mathcal{L}^{t} \boldsymbol{A}=-\omega^{t} \mathcal{R}^{t} \boldsymbol{A} .
$$

The solution of the above equation takes the functional form

$$
\omega^{t}=\omega^{t}\left(k^{t}, \beta, \epsilon, \alpha, B_{v}, F\right) .
$$

In the scheme of spatial linear stability analysis, on the other hand, the angular frequency is real and is denoted as $\omega^{s}=\omega_{r}^{s}$ while the wavenumber is complex as $k^{s}=k_{r}^{s}+\mathrm{i} k_{i}^{s}$, where $k_{r}^{s}$ is the real wavenumber and $k_{i}^{s}$ is the growth rate of the disturbance in space. In the case of a downstream propagating disturbance, which is the case considered herein, $k_{i}^{s}$ assumes negative values. In the scope of spatial analysis, it is convenient to decompose the matrix $\mathcal{M}$ in the form:

$$
\mathcal{M}=\mathcal{L}^{s}+k^{s} \mathcal{R}_{1}^{s}+k^{s 2} \mathcal{R}_{2}^{s},
$$

where the entries of $\mathcal{L}^{s}$ are composed by the terms of the entries of $M$ which do not contain $k^{s}$ (or $k^{s^{2}}$ ), and $\mathcal{R}_{1}$ and $\mathcal{R}_{2}$ are composed by the coefficients of $k^{s}$ and $k^{s^{2}}$, respectively, in the entries of $\mathcal{M}$. Substituting the above decomposition into (22) yields the following nonlinear eigenproblem:

$$
\mathcal{L}^{s} \boldsymbol{A}=-\left(k^{s} \mathcal{R}_{1}^{s}+k^{s 2} \mathcal{R}_{2}^{s}\right) \boldsymbol{A} .
$$

The above expression is linearized in the form

$$
\left(\begin{array}{cc}
\mathcal{L}^{s} & 0 \\
0 & I
\end{array}\right)\left(\begin{array}{c}
\boldsymbol{A} \\
k^{s} \boldsymbol{A}
\end{array}\right)=k^{s}\left(\begin{array}{cc}
-\mathcal{R}_{1}^{s} & -\mathcal{R}_{2}^{s} \\
I & 0
\end{array}\right)\left(\begin{array}{c}
\boldsymbol{A} \\
k^{s} \boldsymbol{A}
\end{array}\right) .
$$


where $I$ is the identity matrix. The solution of the above equation takes the functional form

$$
k^{s}=k^{s}\left(\omega^{s}, \beta, \epsilon, \alpha, B_{v}, F\right) .
$$

\section{RESULTS AND DISCUSSION}

Unless otherwise specified, the parameters $\beta, \epsilon, \alpha, B_{v}$ and $F$ are kept fixed at $\beta=0.05$, $\epsilon=6 \times 10^{-4}, \alpha=10, B_{v}=0.55$ and $F=0.5$. This set of values is based on typical values of the experiments of Ikeda et al. (1991). Also, $N=30$ was adopted in the Chebyshev polynomials, with results independent of the numerical resolution.

\section{Spectral analysis}

In the temporal problem, the eigenvalue $\omega^{t}$ with the largest imaginary part $\omega_{i}^{t}$ is selected to compute the temporal growth rate of perturbation. Fig. 4(a) depicts the temporal spectrum for the case $k_{r}^{t}=5.0$, where there is a single eigenvalue which corresponds to temporal growth $\left(\omega_{i}^{t}>0\right)$, located in the range of positive $\omega_{r}^{t}$. Fig. 4(b) displays the spatial spectrum for the case $\omega_{r}^{s}=$ 3.2. Differently from the temporal spectrum, the spatial spectrum contains eigenvalues in all four quadrants.

Fig. 5 depicts instability diagrams for the temporal case. In Fig. 5(a), the temporal growth rate is positive for the range $0.92 \leq k^{t} \leq 12.78$, which corresponds to the range $0.56 \leq \omega_{r}^{t} \leq 7.53$, as depicted in Fig. 5(b). Therefore, the neutral cases where the imaginary parts of $\omega$ and $k$ are both equal to zero correspond to $(k, \omega)=(0.92,0.56)$ and $(k, \omega)=(12.78,7.53)$. When selecting eigenvalues for computing spatial growth, $k_{r}^{s}$ and $\omega_{r}^{s}$ must then be same ranges of $k_{r}^{t}$ and $\omega_{r}^{t}$ for which temporal growth takes place. Additionally, $k_{i}^{s}$ must be negative because the disturbance propagates downstream.

\section{Evaluation of the perturbation growth}

Following Gaster (1962), the spatial growth rate $k_{i}^{s}$ can be alternatively determined from the temporal analysis as

$$
k_{i}^{s}=-\frac{\omega_{i}^{t}}{c_{g}^{t}},
$$


where $c_{g}=\partial \omega_{r} / \partial k_{r}$ is the group velocity.

Fig. 6 depicts instability diagrams of the spatial growth rate $-k_{i}^{s}$ determined from the temporal and the spatial analyses. The curves of the temporal and spatial analyses are roughly similar, with a small discrepancy observed in the range of large values of $-k_{i}^{s}$. This discrepancy is expected because (31) is obtained assuming that the terms of order $O\left(\max \left(\omega_{i}^{t^{2}}\right)\right)$ are negligible (Gaster 1962). The maximum spatial growth rate in the temporal curve is $-k_{i}^{s}=0.878$ and takes place at $\omega_{r}^{t}=3.76$, while the maximum growth rate in the spatial curve is $-k_{i}^{s}=0.898$ and is located at $\omega_{r}^{s}=3.67$. For comparison, the maximum temporal growth in Fig. $5\left(\omega_{i}^{t}=0.519\right)$ takes place at $\omega_{r}^{t}=3.45$.

Fig. 7 depicts the contours of the spatial growth rate $-k_{i}^{s}$, determined from the temporal and the spatial analyses, in the $\epsilon-k_{r}$ plan. The contours of the neutral instability $\left(k_{i}=0\right)$ are obviously coincident. As the absolute value of the growth rates increase, the discrepancy between the contours obtained from the temporal and the spatial analyses becomes more significant.

The results of this study indicate that the frequencies corresponding to the maximum amplification in space are slightly larger (if not virtually equal) than those corresponding to the maximum amplification in time. Ikeda et al. (1991, 1994), Tsujimoto (1991) and White and Nepf (2007, 2008) performed experiments (Table 1) where vortices were generated in an open-channel featuring a lateral array of regularly spaced pile dikes, which is the same set up assumed in the present formulation. Lima and Izumi (2014a) predicted the period of vortex shedding associated with the maximum amplification in time for the experimental cases of Ikeda et al. $(1991,1994)$ and Tsujimoto (1991). The predicted period was approximately $50 \%$ of the period observed in the experiments, owing to the fact that the linear stability analysis describes the onset of instability while the experimental measurements were obtained for the developed stage of perturbations. The predicted vortex shedding frequencies associated with the maximum growth in space would then be slightly smaller than those from the temporal case, if not virtually equal.

According to Ho and Huerre (1984), the Strouhal number of the most amplified wave in a free shear layer prior to the nonlinear development of instabilities, $\mathrm{St}=\tilde{\omega}_{m} \tilde{\theta} / \tilde{U}_{a}$, takes the nearly con- 
stant value of 0.032 for any hyperbolic tangent base flow profile, where $\tilde{\omega}_{m}$ is the natural frequency of the maximum instability, $\tilde{U}_{a}$ is the average of the far field velocities, $\tilde{U}_{a}=\left(\tilde{U}_{\infty}+\tilde{U}_{-\infty}\right) / 2$, and $\tilde{\theta}$ is the momentum thickness of the undisturbed flow. Ho and Huerre (1984) also noted that the Strouhal number of the most amplified wave in a free shear layer drops significantly below the neutral value of 0.032 as the flow develops and nonlinear interactions become important. Lima and Izumi (2014b) determined the Strouhal number based on the most amplified wave determined from the temporal linear stability analysis and the momentum thickness calculated from the base flow velocity solution (15) for the experimental runs of Ikeda et al. (1991) and White and Nepf (2007, 2008). Results were within the range $0.034 \leq \mathrm{St} \leq 0.042$. In the case of the spatial analysis, the range determined herein is $0.035 \leq \mathrm{St} \leq 0.046$. The slight overestimation from the present formulation in either the temporal or spatial approach may be because the base flow velocity in (15) is set completely free of the transverse motions of the shear layer, while the hyperbolic-tangent velocity profile, for which $\mathrm{St} \approx 0.032$, may have effects of such transverse motions.

\section{Evaluation of the phase velocity}

Fig. 8 depicts curves of the phase velocity $c_{p}=\omega_{r} / k_{r}$ as a function of the frequency $\omega_{r}$ for the temporal and spatial cases and for multiples values of $\beta, \epsilon, \alpha, B_{v}, F$ and $\tilde{B} / \tilde{H}_{0}$.

In Michalke (1964, 1965), the phase velocity in the temporal approach is constant and equal to the group velocity, whereas the phase velocity in the spatial case varies strongly with the frequency $\omega_{r}$, especially for small values of $\omega_{r}$. Michalke (1965) stated that the behavior of the phase velocity in the spatial predictions was observed in the experiments of Freymuth (1965).

In the present work, however, the phase velocity in the temporal problem is not constant, as seen in Fig. 8. A clear discrepancy can be noted between the curves of $c_{p}^{t}$ and $c_{p}^{s}$, mainly in the range of frequencies smaller than that where the spatial and temporal curves intersect. The curves in the temporal and spatial cases intersect near the points where $\omega_{i}$ and $-k_{i}$ are maximized. Therefore, the preferential frequencies for maximum amplification in time and space converge to approximately the same value. Moreover, the values of $c_{p}$ in the temporal and spatial cases at the minimum $\omega_{r}$ for instability tend to converge. This differs remarkably from the results of Michalke 
(1964, 1965). In the present work, the minimum frequency for an unstable flow corresponds to the same phase velocity $c_{p}$, regardless of the approach (spatial or temporal) taken. This is because the base flow is set as the flow completely free from the instabilities due to the shear layer. As the frequency grows, the curves of $c_{p}$ in the spatial and temporal cases often follow distinct paths until they intersect at the vicinity of the frequencies for maximum amplification.

The frequency for maximum amplification increases significantly as the bed friction parameter $\beta$ increases and the kinematic eddy viscosity parameter $\epsilon$ decreases, as depicted in Fig. 8(a) and (b), respectively. Additionally, the discrepancy between $c_{p}$ in the temporal and spatial cases becomes more significant as the value of $\omega_{r}$ at the intersection increases.

The vegetation drag parameter $\alpha$ has a strong effect on $c_{p}$, as depicted in Fig. 8(c). For $\alpha=10^{0}$, the instability is characterized by long waves (small $k_{r}$ ) moderately amplified, as seen in Fig. 9. For $\alpha=10^{1}$, the range of $k_{r}$ for unstable flow reaches higher values; therefore shorter waves are also commonplace as $\alpha$ increases to $10^{1}$. The reduction of $c_{p}$ for $\alpha=10^{2}$ and $10^{3}$ may be attributed to the reduction in the domain of frequencies $\left(\omega_{r}\right)$ for unstable flow.

The discrepancy between $c_{p}$ in the temporal and spatial cases is more significant for larger growth rates. In Fig. 8(b), the discrepancy of the temporal and spatial curves of $c_{p}$ increases with decreasing values of $\epsilon$. As is verified in Fig. 7, the growth rates are larger for smaller values of $\epsilon$. Similarly, for $\alpha=10^{1}$ and $10^{2}$, a greater discrepancy is found in the curves of $c_{p}$ (Fig. 8(c)) and the growth rates reach their maximum range (Fig. 9).

The effect of the non-dimensional vegetated zone width $B_{v}$ on $c_{p}$ is indicated in Fig. 8(d). The curves of $c_{p}$ in the range of small $\omega_{r}$ vary considerably between the temporal and spatial cases and between the values of $B_{v}$. For $\omega_{r}$ larger than the frequency of maximum amplification, the temporal curves of $B_{v}=0.6$ and $B_{v}=1.0$ are virtually coincident. This was also the case for the spatial curves. When the values of $B_{v}$ are sufficiently large, the maximum amplification is virtually independent of $B_{v}$. Because it is assumed that $B_{v}$ is sufficiently large to achieve a constant far field velocity in the vegetated zone, the reliability of the results for $B_{v}=0.2$ is questionable, though it is presented to clarify the tendency of $c_{p}$ at small $B_{v}$. 
The effect of the Froude number $F$ on $c_{p}$ is depicted in Fig. 8(e). For $F=0.001, F=1.0$, and $F=2.0$, the flow is unstable due to the shear layer, and the results show features similar to those from Fig. 8(a-d,f). For $F=3.0$, on the other hand, the flow is unstable due to gravity, and two modes of instability appear, which is verified by the two pairs of curves of $c_{p}$. For $F=3.0$, the temporal and spatial curves are virtually coincident, as are the points where the perturbation growth is maximized.

Finally, the effect of the aspect-ratio $\tilde{B} / \tilde{H}_{0}$ on $c_{p}$ is studied in Fig. 8(f). It is assumed that the variation of the aspect-ratio is solely due to the variation of $\tilde{B}$, such that the parameters $\alpha$ and $F$ are kept fixed at $\alpha=10$ and $F=0.5$, while $\beta$ and $\epsilon$ vary. The parameter $B_{v}$ is kept fixed at $B_{v}=0.55$ assuming that the proportion of the widths on the non-vegetated and vegetated zones does not vary. As the aspect-ratio increases, $\beta$ increases, while $\epsilon$ decreases, resulting in Fig. 8(f) being somewhat similar to Fig. 8(a) and (b).

Fig. 10 depicts the phase velocity $c_{p}$ versus the angular frequency $\omega_{r}$ obtained from employing a base flow velocity modified from Michalke $(1964,1965)$ expressed as

$$
U_{0}(y)=\phi+0.5(1-\phi)[1+\tanh (\eta y)]
$$

instead of the analytical solutions expressed in (15). In the above, $\eta$ is introduced such that the velocity profiles of (15) and (32) have the same shear layer width. The shear layer width is defined based on the analytical solutions (15) as the distance along the transverse direction $y$ from the location where $99 \%$ of the velocity difference $\psi-\phi$ is recovered to the location where $99 \%$ of the velocity difference $1-\psi$ is recovered. The parameter $\eta$ is therefore a function of $\beta$, $\epsilon$ and $\alpha$. In Fig. $10, \beta=0.05$ and $\epsilon=6 \times 10^{-4}$, for which $\eta$ is determined to be 9.55 and 12.13 for $\alpha=10$ and 100, respectively. In Fig. 10(a), the parameters $\beta$ and $\epsilon$ are kept in the perturbation equations (17-18). In Fig. 10(b), $\epsilon$ is dropped from (17-18), while $\beta$ is maintained. In Fig. 10(c), $\beta$ is dropped while $\epsilon$ is maintained and finally, in Fig. 10(d), both $\beta$ and $\epsilon$ are dropped. Fig. 10(d) presents features notably similar to the results of Michalke $(1964,1965)$, such as 1) $c_{p}$ constant and 
equal to $(\phi+1) / 2$ in the temporal case; 2) instability taking place from $\omega_{r} \rightarrow 0$ and 3) $c_{p}$ at the minimum $\omega_{r}$ for instability being remarkably distinct in the temporal and spatial cases. The first and second features are also observed in Fig. 10(c). Therefore, the combined effect of parameters $\beta, \epsilon$ and $\alpha$ causes the results of the present work to be distinct from those of Michalke (1964, 1965). Moreover, the effect of large Froude number $F$ modifies the results considerably, as seen in Fig. 8(e). In Fig. 10(a), although the parameters $\beta$ and $\epsilon$ are kept in the perturbation equations, the curves are distinct from those of Fig. 8(c). In Figs. 10(a) and (b), the flow is stable at small angular frequency $\omega_{r}$ (as well as wavenumber $k$ ) because the bed friction effect, expressed by the parameter $\beta$, dumps small scale perturbations of the shear layer.

\section{Evaluation of the eigenfunction}

Michalke (1964, 1965) and Freymuth (1966) demonstrated that only the spatial analysis can capture the distribution of the disturbance velocity component across the spanwise domain. In particular, the temporal analysis carried out by Michalke (1964) resulted in symmetric and antisymmetric cross-distributions of the real and imaginary parts the eigenfunction, respectively. This behavior was not observed in Michalke's (1965) spatial analysis, where the cross-distribution of the real and imaginary parts of the eigenfunctions were asymmetric. Moreover, a sharp phase reversal of the disturbance velocity component, observed in the experiments of Freymuth (1965), was predicted by the spatial analysis by Michalke (1965), but not in the temporal analysis (Michalke (1964)) except in the case of a neutral disturbance.

Fig. 11 depicts the real and imaginary parts as well as the absolute values of the eigenfunction $U_{1}(y)$ determined in the present work across the spanwise domain. The eigenfunction is normalized such that $U_{1}(0)=1$. The temporal and spatial curves are obtained for the wavenumber and frequency where the amplification is maximized, which correspond to $\left(k^{t}, \omega_{r}^{t}\right)=(5.48,3.45)$ in the temporal case and $\left(k_{r}^{s}, \omega^{s}\right)=(5.91,3.70)$ in the spatial case. The cross-distributions of the eigenfunction $U_{1}(y)$ from both the temporal and spatial analyses are found to be asymmetric and both contain sharp phase reversals at $y \approx-0.1$ and $y \approx 0.1$. This differs from the results reported by Michalke (1965). 


\section{CONCLUSIONS}

Temporal and spatial linear stability analyses of an open-channel flow covered with vegetation at one of its sides are performed. A viscous-flow approach was employed, with the Reynolds stress expressed by means of a kinematic eddy viscosity associated with sub-depth scale turbulences. As a result, a base flow consisting of the flow completely free from the effect of the transverse motions of the shear layer was derived. This is distinct from previous approaches where the base flow consisted of the average flow for which a hyperbolic-tangent velocity distribution was imposed. The average flow is affected by the increased mixing efficiency caused by the development of large scale turbulences in the shear layer. Thus, the results of the formulations used in this study may provide more reliable insight into the onset of instabilities in the shear layer, compared to these previous approaches.

In this work, less difference was found between the results from the temporal and spatial approaches than those from previous inviscid approaches. This may be attributed not only to the inclusion of the effect of the kinematic eddy viscosity in the present formulation, but also to the inclusion of the effects of bed shear, gravity and differential drag in the vegetated zone.

The phase velocity corresponding to the minimum angular frequency for instability was found to be approximately the same in both the temporal and spatial approaches. Moreover, the phase velocity was determined to be correlated to the frequency not only in the spatial case, but also in the temporal case. The frequency of the maximum amplification was found to be slightly smaller in the spatial case than in the temporal case (if not virtually equal). Finally, the cross-distribution of the streamwise velocity eigenfunction was found to be asymmetric for both the temporal and spatial cases.

The most noticeable difference between results from the temporal and spatial analyses may be attributed to the phase velocity in the range of small frequencies (excluding the vicinity of the minimum neutral frequency). This is more clearly observed in the case of small eddy viscosity parameter $\epsilon$ and resembles the discrepancy between results from spatial and temporal linear stability analysis in inviscid shear layers. 


\section{APPENDIX I. REFERENCES}

Carnie, R., Tonina, D., McKean, J. A., and Isaak, D. (2015). "Habitat connectivity as a metric for aquatic microhabitat quality: application to Chinook salmon spawning habitat." Ecohydrology, doi: 10.1002/eco.1696.

Chen, D. and Jirka, G. H. (1997). "Absolute and convective instabilities of plane turbulent wakes in a shallow water layer." Journal of Fluid Mechanics, 338, 157-172.

Chu, V. H., Wu, J.-H., and Khayat, R. E. (1991). "Stability of transverse shear flows in shallow open channels." Journal of Hydraulic Engineering, ASCE, 117(11), 1370-1388.

Fischer, H. B., List, E. J., Koh, R. C. J., Imberger, J., and Brooks, N. H. (1979). Mixing in Inland and Coastal Waters. Academic, New York.

Freymuth, P. (1965). Diss. Technische Universität Berlin (as cited in Michalke, 1965).

Freymuth, P. (1966). “On transition in a separated laminar boundary layer.” Journal of Fluid Mechanics, 25, 683-704.

Gaster, M. (1962). "A note on the relation between the temporally-increasing and spatiallyincreasing disturbances in hydrodynamic instability.” Journal of Fluid Mechanics, 14, 222-224.

Ghidaoui, M. S. and Kolyshkin, A. A. (1999). "Linear stability analysis of lateral motions in compound open channel." Journal of Hydraulic Engineering, ASCE, 125(8), 871-880.

Ho, C.-M. and Huerre, P. (1984). "Perturbed free shear layers." Annual Review of Fluid Mechanics, $16,365-424$.

Ikeda, S., Izumi, N., and Itoh, R. (1991). "Effects of pile dikes on flow retardation and sediment transport." Journal of Hydraulic Engineering, ASCE, 117(11), 1459-1478.

Ikeda, S., Ohta, K., and Hasegawa, H. (1994). "Instability-induced horizontal vortices in shallow open-channel flows with an inflection point in skewed velocity profile." Journal of Hydroscience and Hydraulic Engineering, 12, 69-84.

Kitamura, T., Jia, Y., Tsujimoto, T., and Wang, S. (1998). "Sediment transport capacity in channels with vegetation zone." Water Resource Engineering '98, S. R. Abt, J. Youngpezeshk, and C. C. Watson, eds., number 326, Baltimore, ASCE Publications. 
Kolyshkin, A. A. and Ghidaoui, M. S. (2002). "Gravitational and shear instabilities in compound and composite channels." Journal of Hydraulic Engineering, ASCE, 128(12), 1076-1086.

Lima, A. C. and Izumi, N. (2014a). "Linear stability analysis of open-channel shear flow generated by vegetation.” Journal of Hydraulic Engineering, ASCE, 140(3), 231-240.

Lima, A. C. and Izumi, N. (2014b). "On the nonlinear development of shear layers in partially vegetated channels." Physics of Fluids, 26, 084109-1-084109-22.

Michalke, A. (1964). “On the inviscid instability of hyperbolic tangent velocity profile.” Journal of Fluid Mechanics, 19, 543-556.

Michalke, A. (1965). "On spatially growing disturbances in an inviscid shear layer." Journal of Fluid Mechanics, 23, 521-544.

Monkewitz, P. A. and Huerre, P. (1982). "Influence of the velocity ratio on the spatial instability of mixing layers." Physics of Fluids, 25, 1137-1143.

Nadaoka, K. and Yagi, H. (1998). "Shallow-water turbulence modeling and horizontal large-eddy computation of river flow." Journal of Hydraulic Engineering, ASCE, 124(5), 493-500.

Prooijen, B. C. and Uijttewaal, W. S. J. (2002). "A linear approach for the evolution of coherent structures in shallow mixing layers." Physics of Fluids, 14, 4105-4114.

Schmid, P. J. and Henningson, D. S. (2001). Stability and transition in shear flows. Springer.

$\mathrm{Su}, \mathrm{X}$. and Li, C. W. (2002). "Large eddy simulation of free surface turbulent flow in partially vegetated open channels." International Journal of Numerical Methods in Fluids, 39, 919-937.

Tamai, N., Asaeda, T., and Ikeda, H. (1986). "Study on generation of periodical large surface eddies in a composite channel flow." Water Resources Research, 22(7), 1129-1138.

Tsujimoto, T. (1991). “Open channel flow with bank vegetation.” KHL Communication, 2, 41-54.

Tsujimoto, T. and Kitamura, T. (1995). "Lateral bed-load transport and sand-ridge formation near vegetation zone in an open channel." Journal of Hydroscience and Hydraulic Engineering, 3545.

Uijttewaal, W. S. J. and Booij, R. (2000). "Effects of shallowness on the development of freesurface mixing layers." Physics of Fluids, 12, 4392-4402. 
White, B. L. and Nepf, H. M. (2007). "Shear instability and coherent structures in shallow flow adjacent to a porous layer." Journal of Fluid Mechanics, 593, 1-32.

White, B. L. and Nepf, H. M. (2008). "A vortex-based model of velocity and shear stress in a partially vegetated shallow channel." Water Resources Research, 44, W01412, doi:10.1029/2006WR005651. 
The following symbols are used in this paper:

$$
\begin{aligned}
& A=\text { amplitude; } \\
& a=\text { vegetation density parameter; } \\
& B=\text { non-vegetated zone width; } \\
& B_{v}=\text { vegetated zone width; } \\
& C_{d}=\text { vegetation drag coefficient; } \\
& C_{f}=\text { bed friction coefficient; } \\
& c_{g}=\text { group velocity; } \\
& c_{p}=\text { phase velocity; } \\
& D_{x}, D_{y}=\text { streamwise and transverse vegetation drag components, respectively; } \\
& d=\text { diameter of cylinders; } \\
& F=\text { Froude number; } \\
& g=\text { gravity acceleration; } \\
& H=\text { flow depth; } \\
& k=\text { wavenumber; } \\
& l_{x}, l_{y}=\text { distances between the center of two adjacent cylinders in the } x \text { and } y \text { directions; } \\
& S=\text { streamwise bed slope of the channel; } \\
& T_{b x}, T_{b y}=\text { streamwise and transverse bed shear stress components, respectively; } \\
& T_{j}(\xi), T_{j}(\zeta)=\text { Chebyshev polynomials in } \xi \text { and } \zeta \text { of degree } j \text {; } \\
& t=\text { time; } \\
& U, V=\text { streamwise and transverse velocities, respectively; } \\
& U_{0}=\text { base state flow velocity; } \\
& U_{1}, V_{1}, H_{1}=\text { eigenfunctions; } \\
& U_{f}=\text { friction velocity; } \\
& x, y=\text { streamwise and transverse coordinates, respectively; } \\
& \alpha=\text { vegetation drag and density parameter; }
\end{aligned}
$$




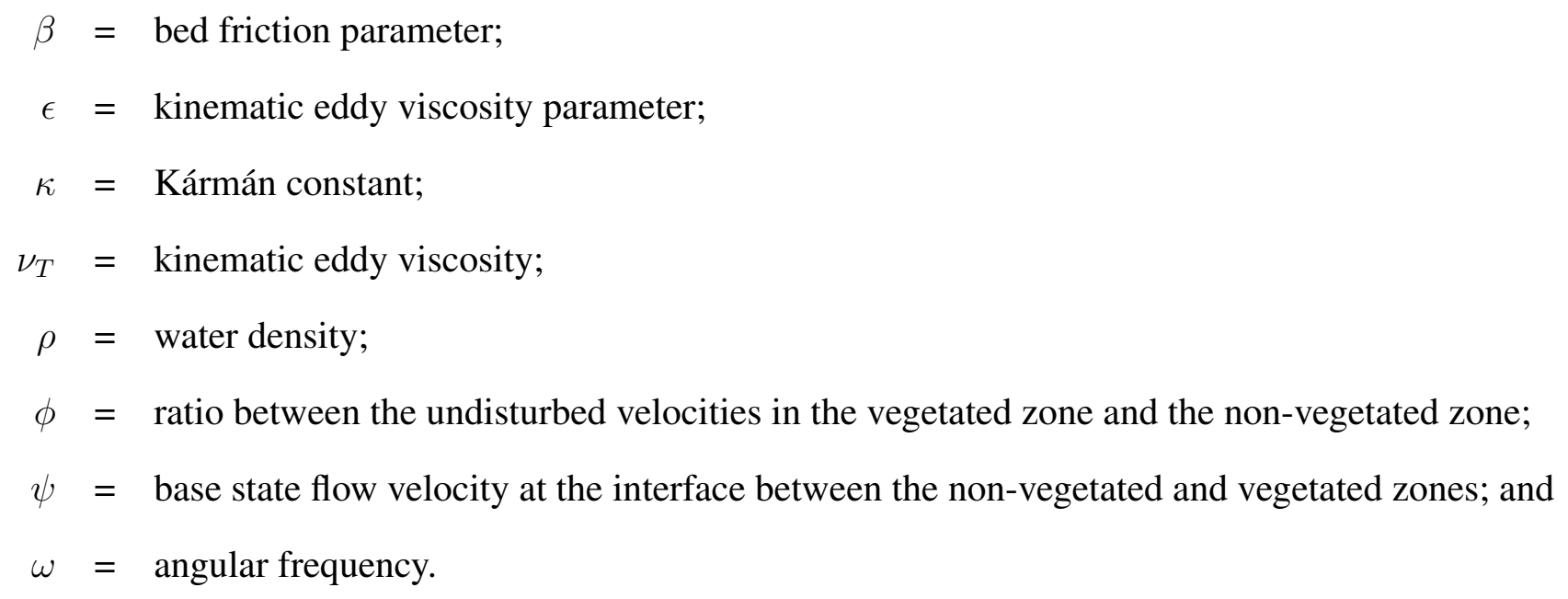

$\infty,-\infty=$ far field in the non-vegetated and vegetated zones, respectively. 


\section{List of Tables}

1 Hydraulic parameters for the experiments of Ikeda et al. (1991, 1994) (runs 1-5), Tsujimoto (1991) (runs IW1-IW3) and White and Nepf (2007, 2008) (runs I-XI). . 27 
TABLE 1: Hydraulic parameters for the experiments of Ikeda et al. (1991, 1994) (runs 1-5), Tsujimoto (1991) (runs IW1-IW3) and White and Nepf (2007, 2008) (runs I-XI).

\begin{tabular}{crrrrrrrrrrr}
\hline \hline Run & $\begin{array}{c}\text { Discharge } \\
(1 / \mathrm{s})\end{array}$ & $\begin{array}{c}\tilde{U}_{\infty} \\
(\mathrm{cm} / \mathrm{s})\end{array}$ & $\begin{array}{c}\tilde{U}_{f} \\
(\mathrm{~cm} / \mathrm{s})\end{array}$ & $\begin{array}{c}\tilde{H} \\
(\mathrm{~cm})\end{array}$ & \multicolumn{1}{c}{$\begin{array}{c}C_{d} \tilde{a} \\
\left(\mathrm{~cm}^{-1}\right)\end{array}$} & & $\begin{array}{c}\epsilon \\
\left(\times 10^{-4}\right)\end{array}$ & $\alpha$ & $B_{v}$ & $F$ \\
\hline 1 & 15.3 & 39.5 & 3.08 & 6.0 & 0.0010 & 0.020 & 0.061 & 5.19 & 9.87 & 0.57 & 0.51 \\
2 & 22.6 & 58.8 & 4.58 & 6.0 & 0.0033 & 0.020 & 0.061 & 5.19 & 9.87 & 0.57 & 0.77 \\
3 & 18.6 & 51.8 & 4.18 & 5.0 & 0.0033 & 0.018 & 0.079 & 4.48 & 6.89 & 0.57 & 0.74 \\
4 & 7.0 & 33.7 & 3.04 & 3.0 & 0.0033 & 0.020 & 0.164 & 3.00 & 3.69 & 0.57 & 0.62 \\
5 & 24.0 & 43.7 & 3.19 & 8.5 & 0.00067 & 0.020 & 0.038 & 6.88 & 16.0 & 0.57 & 0.48 \\
IW1 & - & 41.6 & 2.31 & 3.7 & 0.0015 & - & 0.024 & 4.84 & 67.9 & 0.43 & 0.69 \\
IW2 & - & 47.5 & 3.05 & 3.8 & 0.0025 & - & 0.030 & 5.84 & 50.8 & 0.43 & 0.78 \\
IW3 & - & 57.0 & 3.34 & 3.9 & 0.0029 & - & 0.025 & 5.40 & 63.5 & 0.43 & 0.93 \\
I & - & 17.7 & 1.81 & 6.8 & - & 0.092 & 0.058 & 3.99 & 63.0 & 0.50 & 0.22 \\
II & - & 21.7 & 2.27 & 10.4 & - & 0.092 & 0.023 & 4.73 & 154 & 0.50 & 0.21 \\
III & - & 24.0 & 2.67 & 13.8 & - & 0.092 & 0.023 & 7.31 & 160 & 0.50 & 0.21 \\
IV & - & 17.4 & 2.06 & 6.6 & - & 0.285 & 0.064 & 4.02 & 192 & 0.50 & 0.22 \\
V & - & 3.8 & 0.35 & 5.3 & - & 0.242 & 0.061 & 2.82 & 232 & 0.50 & 0.05 \\
VI & - & 12.3 & 1.48 & 6.0 & - & 0.255 & 0.043 & 2.86 & 214 & 0.50 & 0.16 \\
VII & - & 16.8 & 1.93 & 6.6 & - & 2.43 & 0.055 & 3.73 & 1530 & 0.50 & 0.21 \\
VIII & - & 5.9 & 0.44 & 5.5 & - & 2.74 & 0.126 & 4.29 & 1520 & 0.50 & 0.08 \\
IX & - & 9.1 & 0.84 & 6.8 & - & 2.04 & 0.089 & 4.95 & 1310 & 0.50 & 0.11 \\
X & - & 29.6 & 3.44 & 7.8 & - & 1.77 & 0.066 & 5.22 & 1100 & 0.50 & 0.34 \\
XI & - & 22.0 & 2.51 & 13.9 & - & 2.43 & 0.032 & 8.70 & 2880 & 0.50 & 0.19 \\
\hline \hline & & & & & & & & & & \\
\hline
\end{tabular}




\section{List of Figures}

1 Conceptual diagram of the channel with vegetation. (a) The cross-sectional view, and (b) the plan view. . . . . . . . . . . . . . . . . 30

2 Lateral distribution of the base flow velocity $U_{0}$ for the case $\beta=0.05, \alpha=10$ and variable sub-depth eddy viscosity parameter $\epsilon \ldots \ldots$. . . . . . . . . 31

3 Base state velocity $U_{0}$ (continuous line) and measured streamwise velocity $U$ at the developed state of perturbations along the lateral direction $y$, for run 1 of Ikeda et al. (1994), where $\beta=0.061, \epsilon=5.2 \times 10^{-4}$ and $\alpha=9.9$. . . . . . . . 32

4 (a) Temporal spectrum for $k^{t}=5.0$ and (b) spatial spectrum for $\omega^{s}=3.2$. The arrows indicate the eigenvalues to be selected for computing the perturbation growth. 33

$5 \quad$ Instability diagrams for the temporal case: (a) $\omega_{i}^{t}$ versus $k^{t}$; (b) $\omega_{i}^{t}$ versus $\omega_{r}^{t}$. . . . 34

6 Spatial growth rates $-k_{i}^{s}$ obtained from the temporal analysis (dashed line) and the spatial analysis (continuous line). . . . . . . . . . . . . . 35

7 Contours of the spatial growth rate $-k_{i}^{s}$ determined from (a) the temporal analysis (dashed lines) and (b) the spatial analysis (continuous lines) in the $\epsilon-k_{r}$ plane. . . . 36

8 Phase velocity $c_{p}$ versus angular frequency $\omega_{r}$ for the temporal analysis (dashed lines) and the spatial analysis (continuous lines). The phase velocity and angular frequency at the maximum amplification are indicated by $\square$ for the temporal analysis and by $\mathbf{\square}$ for the spatial analysis. . . . . . . . . . . . 37

9 Contours of $\omega_{i}$ (dashed lines) and $-k_{i}$ (continuous lines) in the (a) $\alpha-k_{r}$ plane and

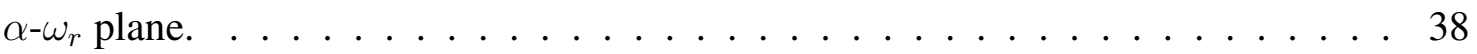

10 Phase velocity $c_{p}$ versus angular frequency $\omega_{r}$ for the temporal analysis (dashed lines) and the spatial analysis (continuous lines) calculated using the base flow velocity of equation (32). (a) Perturbation equations (17-18) not modified; (b) $\epsilon$ dropped from (17-18); (c) $\beta$ dropped from (17-18); (d) $\beta$ and $\epsilon$ dropped from

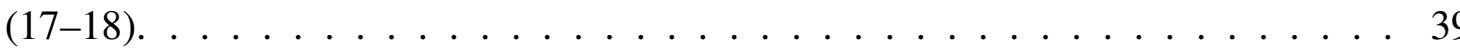


11 Lateral distribution of the (a) real part, (b) imaginary part and (c) absolute value of eigenfunction $U_{1}(y)$ at the maximum amplification in time (dashed curves) and space (continuous curves). . . . . . . . . . . . . . . . . . 40 


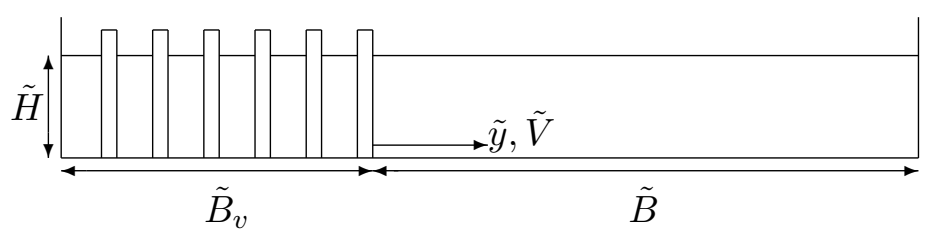

(a)

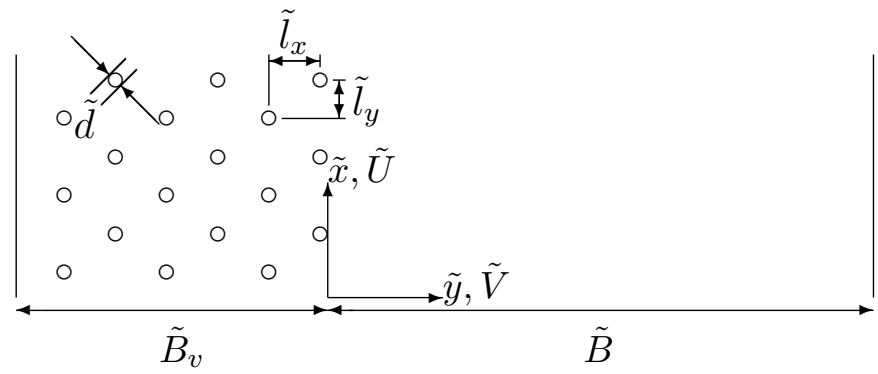

(b)

FIG. 1: Conceptual diagram of the channel with vegetation. (a) The cross-sectional view, and (b) the plan view. 


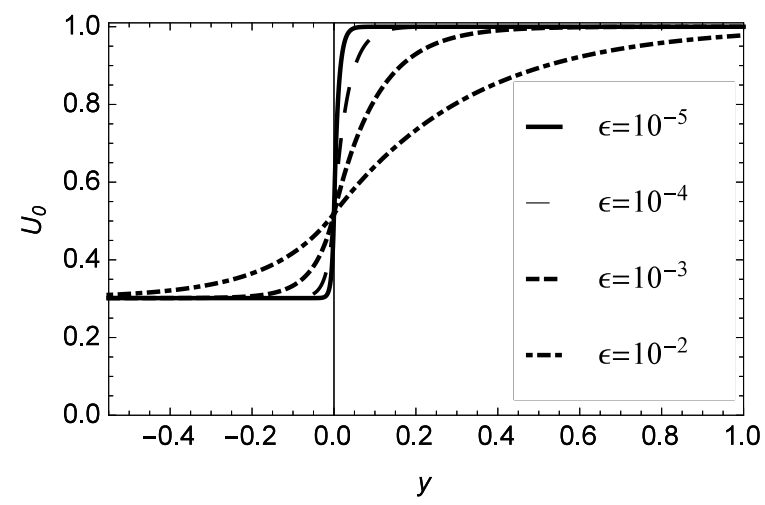

FIG. 2: Lateral distribution of the base flow velocity $U_{0}$ for the case $\beta=0.05, \alpha=10$ and variable sub-depth eddy viscosity parameter $\epsilon$. 


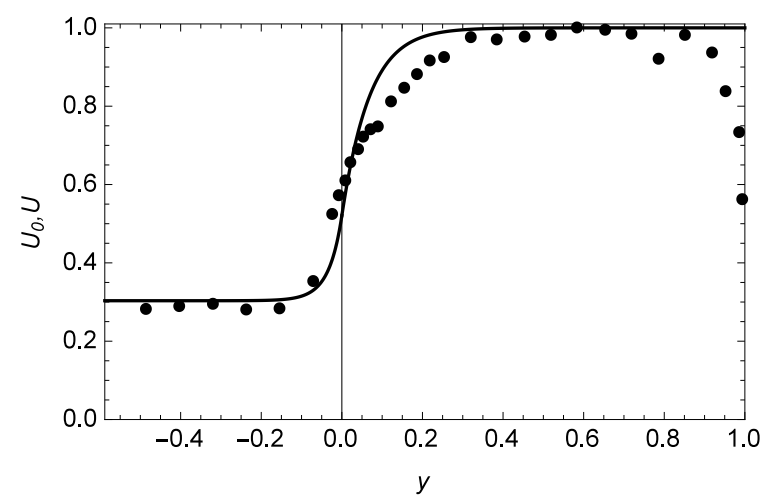

FIG. 3: Base state velocity $U_{0}$ (continuous line) and measured streamwise velocity $U$ at the developed state of perturbations along the lateral direction $y$, for run 1 of Ikeda et al. (1994), where $\beta=0.061, \epsilon=5.2 \times 10^{-4}$ and $\alpha=9.9$. 


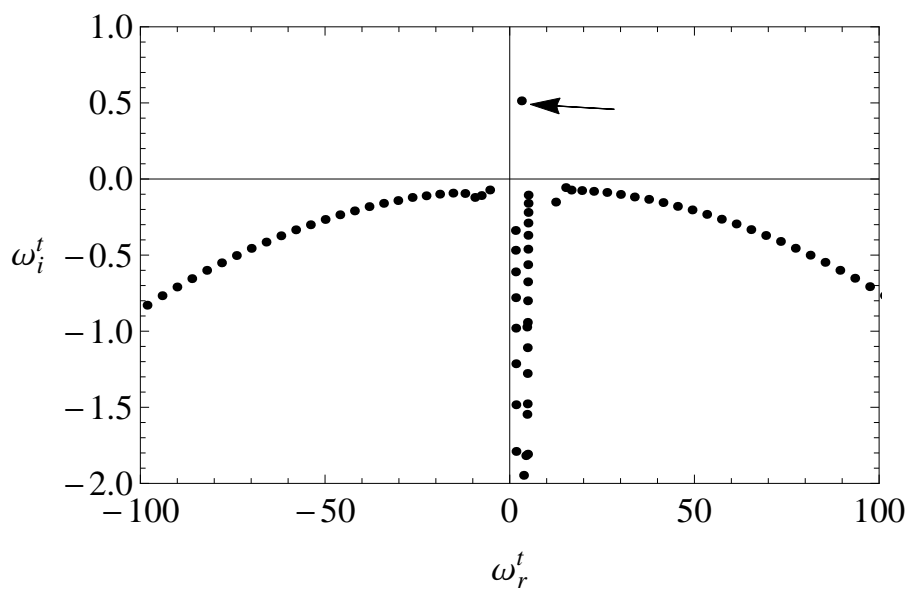

(a)

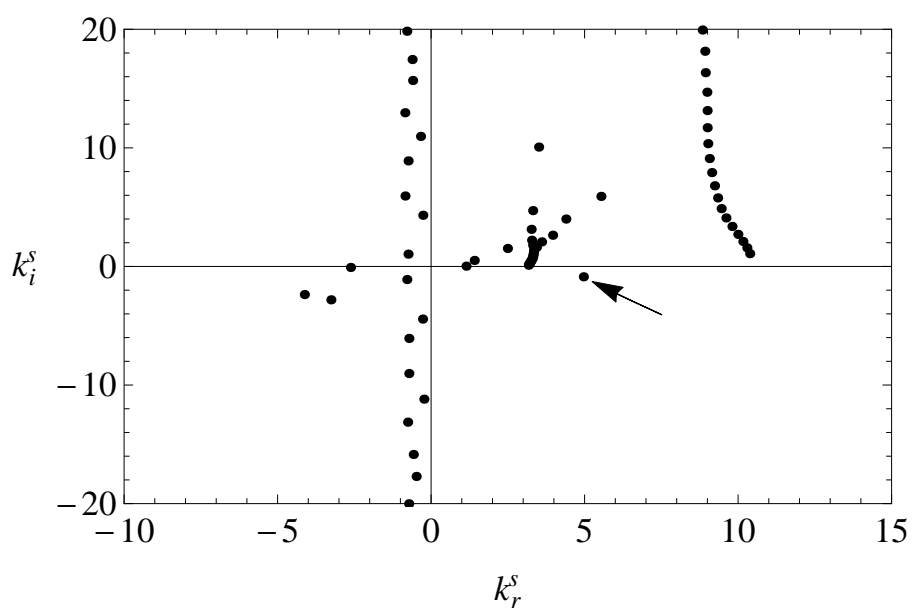

(b)

FIG. 4: (a) Temporal spectrum for $k^{t}=5.0$ and (b) spatial spectrum for $\omega^{s}=3.2$. The arrows indicate the eigenvalues to be selected for computing the perturbation growth. 


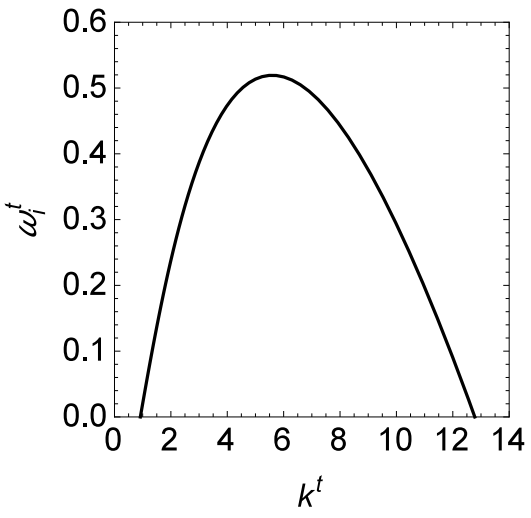

(a)

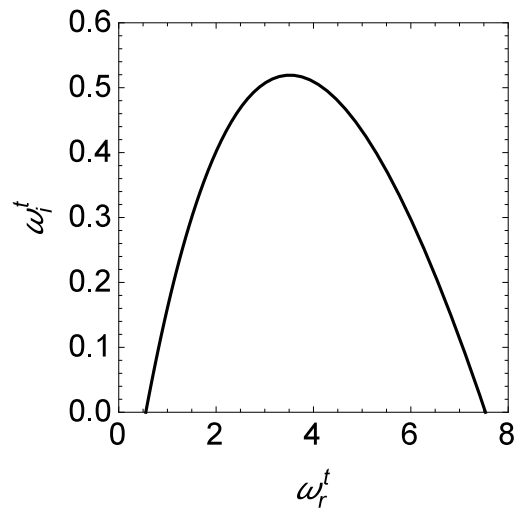

(b)

FIG. 5: Instability diagrams for the temporal case: (a) $\omega_{i}^{t}$ versus $k^{t}$; (b) $\omega_{i}^{t}$ versus $\omega_{r}^{t}$. 


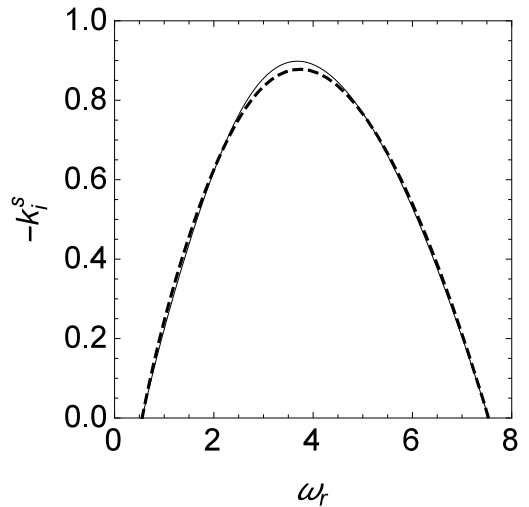

FIG. 6: Spatial growth rates $-k_{i}^{s}$ obtained from the temporal analysis (dashed line) and the spatial analysis (continuous line). 


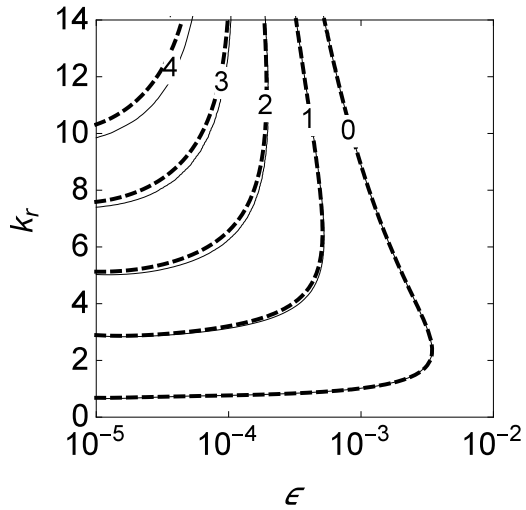

FIG. 7: Contours of the spatial growth rate $-k_{i}^{s}$ determined from (a) the temporal analysis (dashed lines) and (b) the spatial analysis (continuous lines) in the $\epsilon-k_{r}$ plane. 


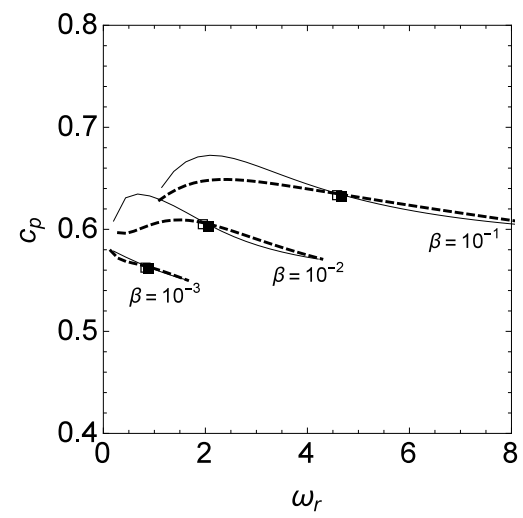

(a)

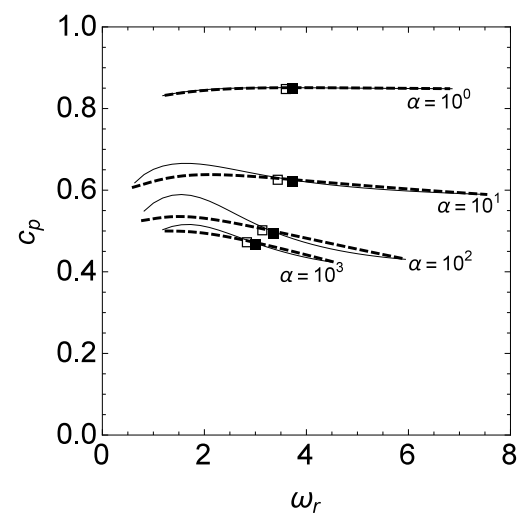

(c)

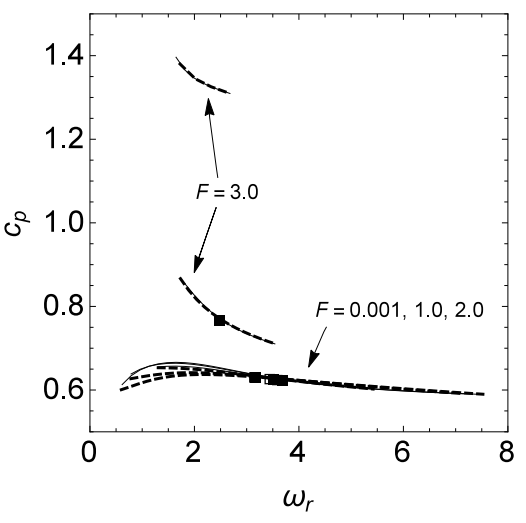

(e)

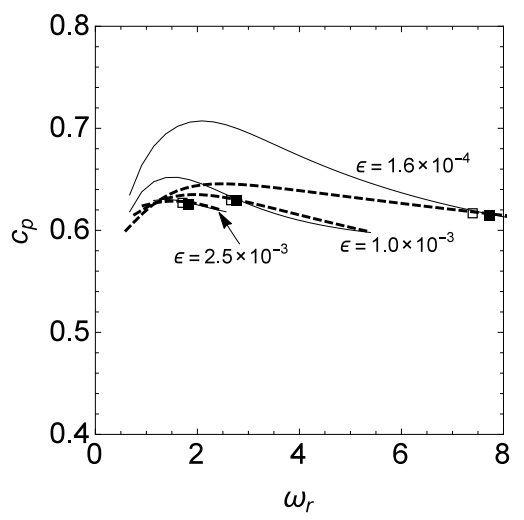

(b)

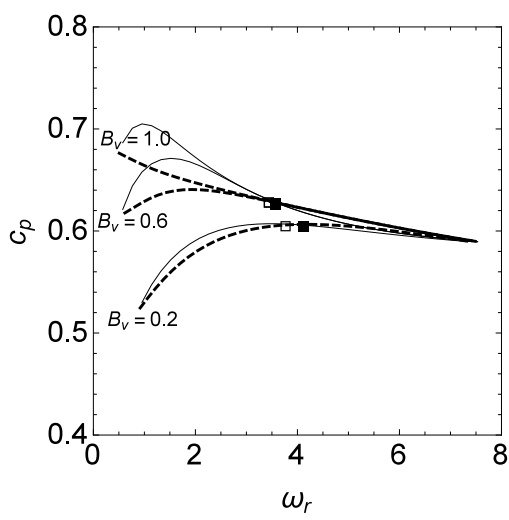

(d)

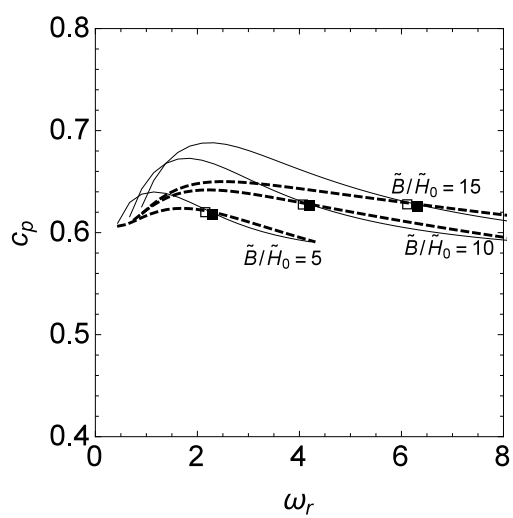

(f)

FIG. 8: Phase velocity $c_{p}$ versus angular frequency $\omega_{r}$ for the temporal analysis (dashed lines) and the spatial analysis (continuous lines). The phase velocity and angular frequency at the maximum amplification are indicated by $\square$ for the temporal analysis and by $\mathbf{\square}$ for the spatial analysis. 


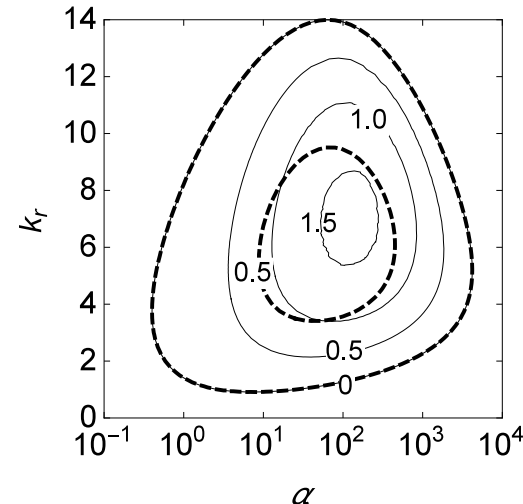

(a)

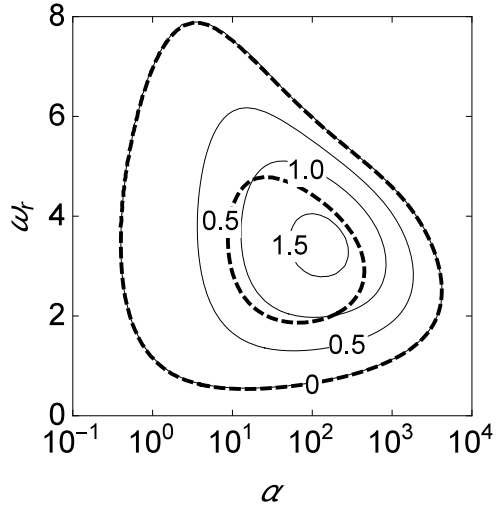

(b)

FIG. 9: Contours of $\omega_{i}$ (dashed lines) and $-k_{i}$ (continuous lines) in the (a) $\alpha-k_{r}$ plane and $\alpha-\omega_{r}$ plane. 


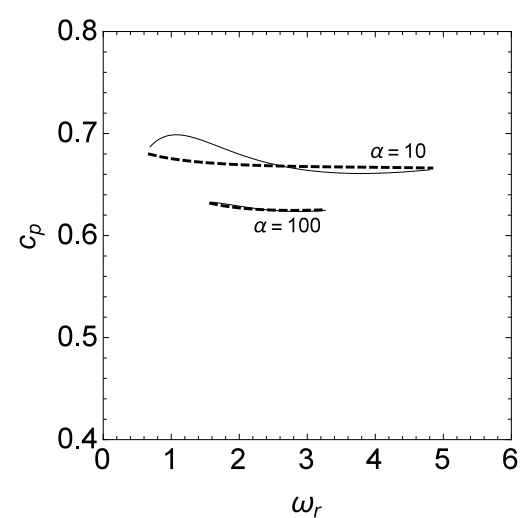

(a)

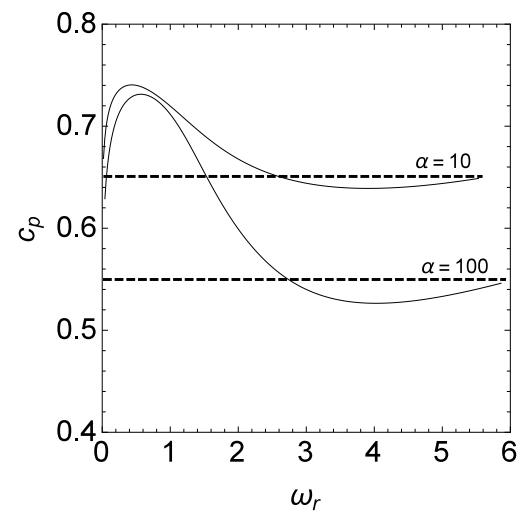

(c)

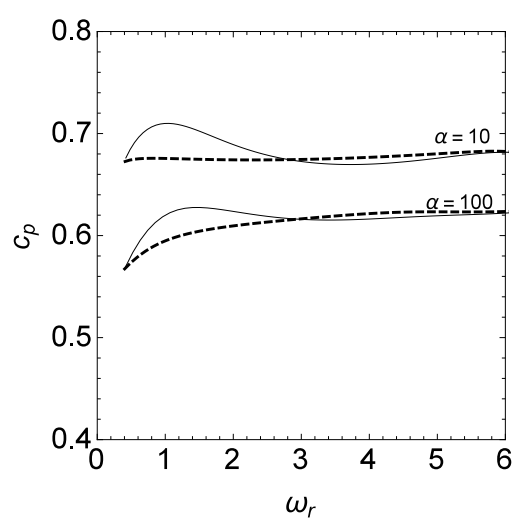

(b)

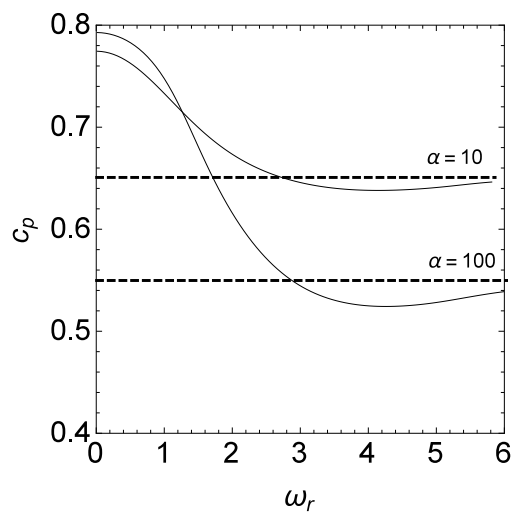

(d)

FIG. 10: Phase velocity $c_{p}$ versus angular frequency $\omega_{r}$ for the temporal analysis (dashed lines) and the spatial analysis (continuous lines) calculated using the base flow velocity of equation (32). (a) Perturbation equations (17-18) not modified; (b) $\epsilon$ dropped from (17-18); (c) $\beta$ dropped from (17-18); (d) $\beta$ and $\epsilon$ dropped from (17-18). 


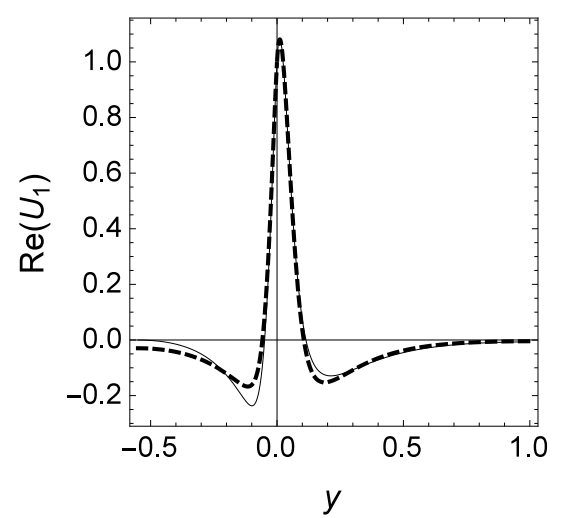

(a)

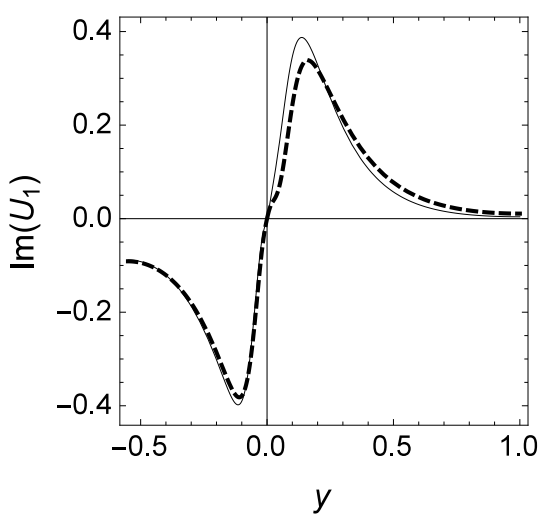

(b)

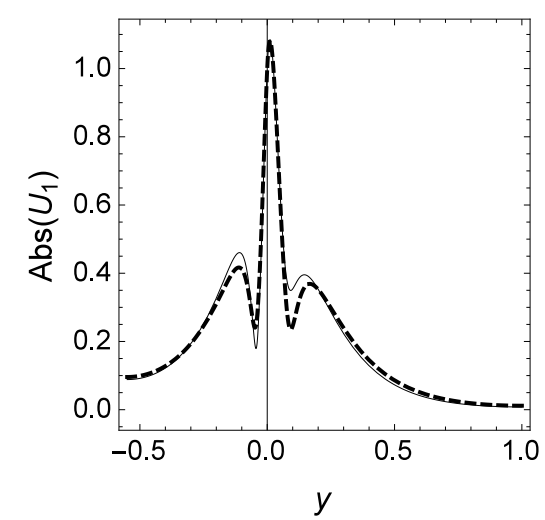

(c)

FIG. 11: Lateral distribution of the (a) real part, (b) imaginary part and (c) absolute value of eigenfunction $U_{1}(y)$ at the maximum amplification in time (dashed curves) and space (continuous curves). 